\title{
Physically consistent metrics with independent parameters instead of standard metrics with unphysical consequences. 2. Rotating sources
}

\author{
Zahid Zakir ${ }^{1}$
}

\begin{abstract}
The Kerr metric, the external metric for a rotating body, contains the equatorial gravitational radius implicitly depending on the specific angular momentum (SAM). Ignoring this dependence due to the formal mathematical approach without understanding the physical aspects led to absurd unphysical consequences in the black hole theory, in particular, that an increase in the rotational energy at increasing SAM weakens gravity, decreasing the gravitational radius at the pole and the effects of gravity (redshifts, mean radii of orbits and shadows). This shortcoming of the Kerr metric is improved in a new form of this metric with an independent parameter - the gravitational radius at the pole, determined by the mass of matter without rotational energy. The contributions of the energies of matter and rotation have the same sign and an increase in SAM strengthens gravity, increasing its effects (the equatorial gravitational radius, redshifts, mean radii of orbits and shadows). The modified form of the Kerr metric describes the gravitational field of a frozar having angular momentum, a star with frozen structure and the surface asymptotically tending to the local gravitational radius (minimal at pole and maximal at equator). The application of this method to the Kerr-Newman metric, including the charge, and to the NUT metric, gave modified forms of these metrics with independent parameters. In the frozar theory, particle energies are positive everywhere, and the theory is free from the non-physical effects of the former black hole theory (horizons, singularities, ergosphere and the extraction of energy from it, evaporation). Thermodynamics of frozars follows from the almost irreversible freezing, as the result of which, during accretion and other processes, the mass of neutral matter without rotational energy grows almost irreversibly.
\end{abstract}

Keywords: Kerr metric, Kerr-Newman metric, NUT metric, frozar, black hole, effects of gravity, redshift, shadow, light deflection, gravitational collapse, frozar thermodynamics

\section{Content}

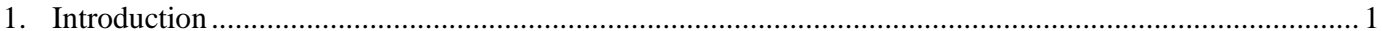

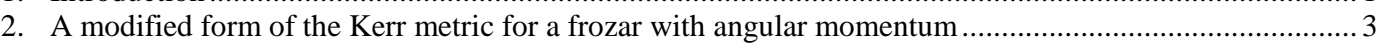

3. Some observable consequences: mean radii of orbits......................................................................... 6

4. A modified form of the Kerr-Newman metric for a charged frozar with angular momentum ....................... 9

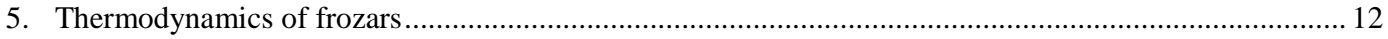

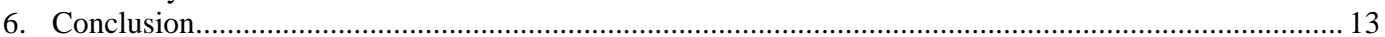

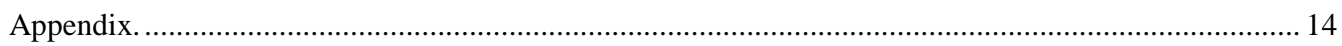

A. Formation of an ellipsoidal frozar at freezing of the rotating star's surface …................................... 14

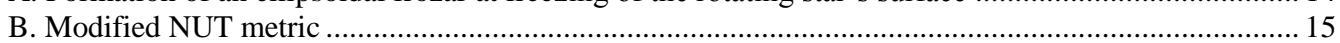

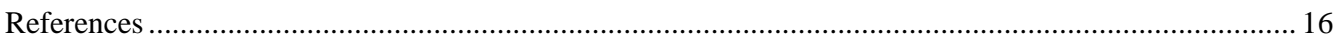

\section{Introduction}

In the first article [1], a new method of modification of standard metrics was formulated, when they contain two or more parameters, but one of them implicitly depends on others. The method allows one to transform the metrics to a new form with independent parameters. In [1], this method was applied to the external metric for the charged dust ball and to the ReissnerNordström (RN) metric and they were transformed into metrics with independent parameters.

${ }^{1}$ Center for Theoretical Physics and Astrophysics, Tashkent Uzbekistan, zzakir@qgph.org, ORCID 
In this case, the dependences of the observable effects of gravity on the charge, predicted in the black hole theory on the basis of the RN metric, turned out to be erroneous and opposite to the physically consistent predictions following from the new metrics for a ball and frozar, in which the dependence of one of the former parameters on the other was taken into account correctly.

In this second article, the same modification of the external metric for the sources with angular momentum $J=M a c$ will be performed and modified forms of the Kerr [2,3] and Kerr-Newman metrics [4] with independent parameters will be obtained. Then the analogous modified form of the NUT metric [5] also will be presented.

In contrast to the spherical sources, in the case of rotating ones, an increase in the rotational energy from the pole to the equator leads to an increase in the local gravitational radius, at which the time component of the metric vanishes and the processes freeze in terms of world time $t$. This radius is minimal at the pole and reaches its maximum at the equator (in the black hole theory, it was interpreted as the outer boundary of the ergosphere).

However, the standard form of the Kerr metric [3,6,7] contains the gravitational radius at the equator, which is determined by the total mass at infinity, including the rotational energy and therefore depending on the specific angular momentum (SAM) $a=J / m c$. In the KerrNewman metric, the total mass also depends on the charge $Q$. Such a dependent nature of one of the parameters was a serious problem hidden in these metrics, and ignoring of this dependence would lead to unphysical consequences.

This is exactly what happened in the black hole theory, where such unphysical consequences, due to the formal mathematical approach without understanding the physical aspects, were seriously considered as predictions for physical effects. In particular, it was argued that the gravity of the black hole is weakening, and the observable effects of gravity, such as redshifts, orbital and shadow radii, decrease with increasing $a$ and $Q$ [6,7]. It turned out that the rest energy gravitates, while other two forms of energy, the rotation energy and the electrostatic energy, antigravitate, which is physically unacceptable.

In the present article, the above mentioned problem of the Kerr and Kerr-Newman metrics is solved by the new method, described in [1], i.e. by transforming them to a modified form, when the new metrics contain only independent parameters. In both cases, the new metrics includes the gravitational radius at the pole $r_{g P}$, determined by the mass of neutral and nonrotating matter and for this reason is independent on $a$ and $Q$.

In these metrics with independent parameters, the contributions of the energies of matter, rotation and electric field have the same sign and therefore the growth of $a$ and/or $Q$, adding the energies of rotation and/or the electric field, strengthens the gravity and its effects, in particular, increases redshifts, the mean radii of orbits and shadows. Thus, the new metrics lead to physically correct dependences of the observable effects on $a$ and $Q$.

The metric for a frozar (an object with a gravitationally frozen structure) $[1,9,10]$ follows from the modified metrics as the surface radius tends to the local gravitational radius, which is minimal at the poles and maximal at the equator. In the frozar theory the energy of particles is positive and the theory is free of the unphysical effects of the black hole theory (singularities, horizons, ergosphere and the extraction of energy from it, evaporation). The thermodynamics of frozars follows from an almost irreversible increase in the frozar's mass due to the practically irreversible gravitational freezing of the falling on the frozar matter and radiation.

In Sections 2 and 4 a modified form of the Kerr and Kerr-Newman metrics are derived, and in Section 3 the results for characteristic radii and their plots are presented. In Section 5 the thermodynamics of frozars is formulated. In the Appendix a proof of the freezing of the frozar surface and a modification of the NUT metric are presented. 


\section{A modified form of the Kerr metric for a frozar with angular momentum}

The space-time interval outside the rotating source in terms of world time $t$ has the form:

$$
d s^{2}=g_{00} c^{2} d t^{2}+g_{11} d r^{2}+g_{22} d \theta^{2}+g_{33} d \varphi^{2}+2 g_{03} c d t d \varphi,
$$

where the components of the metric depend only on $r$ and $\theta$. Let us introduce the notations:

$$
\rho^{2}=r^{2}+a^{2} \cos ^{2} \theta, \quad \Delta(r)=r^{2}-r_{g E} r+a^{2},
$$

where $r_{g E}$ is the gravitational radius at the equator, at which $g_{00}$ vanishes (Figs. 1-2):

$$
g_{00}\left(r_{g E}, \pi / 2\right)=0 \text {. }
$$

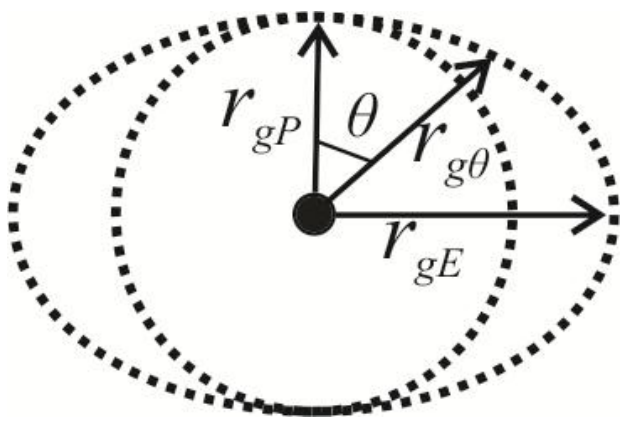

Fig. 1. Structure of the gravitational field of a neutral point source (black hole) with angular momentum.

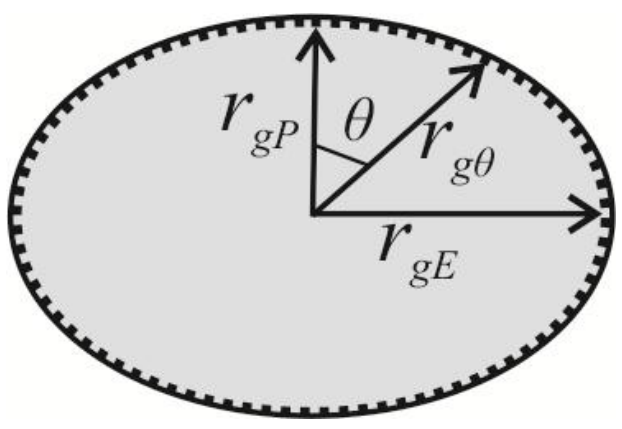

Fig. 2. The surface of a neutral frozar with angular momentum freezes above the gravitational radius by forming an oblate ellipsoid.

Then a line element with the standard form of the Kerr metric [2] (in the Boyer-Lindquist coordinates [3]) has the form (see [6,7]):

$$
\begin{aligned}
d s^{2} & =\left(1-\frac{r_{g E} r}{\rho^{2}}\right) c^{2} d t^{2}-\frac{\rho^{2}}{\Delta} d r^{2}-\rho^{2} d \theta^{2}- \\
& -\left(r^{2}+a^{2}+\frac{r_{g E} r a^{2}}{\rho^{2}} \sin ^{2} \theta\right) \sin ^{2} \theta d \varphi^{2}+\frac{2 r_{g E} r a c}{\rho^{2}} \sin ^{2} \theta d \varphi d t .
\end{aligned}
$$

On the equatorial plane ( $\theta=\pi / 2, \rho^{2}=r^{2}$ ) the line element (4) has the form:

$$
d s^{2}=\left(1-\frac{r_{g E}}{r}\right) c^{2} d t^{2}-\frac{r^{2}}{\Delta} d r^{2}-\left(r^{2}+a^{2}+\frac{r_{g E} a^{2}}{r}\right) d \varphi^{2}+\frac{2 r_{g E} a c}{r} d \varphi d t,
$$

where it is clear that the condition (3), determining the physical meaning of $r_{g E}$, is satisfied.

At the pole ( $\left.\theta=0, \rho^{2}=r^{2}+a^{2}\right)$ the line element (4) has the form:

$$
d s^{2}=\frac{\Delta}{r^{2}+a^{2}} c^{2} d t^{2}-\frac{r^{2}+a^{2}}{\Delta} d r^{2} .
$$

An expression for the gravitational radius at the pole $r_{g P}$ follows from the condition: 


$$
g_{00}\left(r_{g P}, 0\right)=\frac{\Delta\left(r_{g P}\right)}{r_{g P}^{2}+a^{2}}=0, \quad \Delta\left(r_{g P}\right)=r_{g P}^{2}-r_{g E} r_{g P}+a^{2}=0 .
$$

Dividing by $r_{g P}$ the last expression for $\Delta\left(r_{g P}\right)$, we can express $r_{g E}$ in terms of $r_{g P}$ :

$$
r_{g E}=r_{g P}+\frac{a^{2}}{r_{g P}}
$$

It is clearly from (8) that $r_{g E}$ contains, in addition to $r_{g P}$, a contribution of the rotational energy, proportional $a^{2}$, and $r_{g E} \rightarrow r_{g P}$ at $a \rightarrow 0$. Thus, $r_{g E}$ grows quadratically with $a$, while $r_{g P}$ is independent on $a$ (neglecting a weak indirect dependence). Therefore, a modification of the Kerr metric (4) is reduced to the replacement of the dependent parameter $r_{g E}(a)$ by an independent parameter $r_{g P}$ by using the relation (8).

Then for $\Delta$, instead of (2) with $r_{g E}$, we now obtain another expression in terms of $r_{g P}$ :

$$
\Delta=r^{2}-\left(r_{g P}+\frac{a^{2}}{r_{g P}}\right) r+a^{2}=r^{2}\left(1-\frac{r_{g P}}{r}\right)\left(1-\frac{a^{2}}{r_{g P} r}\right),
$$

and the line element at the pole (6) takes the form:

$$
d s^{2}=\frac{r^{2}}{\bar{\rho}^{2}}\left(1-\frac{r_{g P}}{r}\right)\left(1-\frac{a^{2}}{r_{g P} r}\right) c^{2} d t^{2}-\frac{\bar{\rho}^{2}}{r^{2}}\left(1-\frac{r_{g P}}{r}\right)^{-1}\left(1-\frac{a^{2}}{r_{g P} r}\right)^{-1} d r^{2} .
$$

Here $\bar{\rho}^{2}=r^{2}+a^{2}$ and the role of $r_{g P}$ as the gravitational radius is clearly expressed.

At substituting (8) into (5), the line element on the equatorial plane takes the form:

$$
\begin{aligned}
d s^{2} & =\left(1-\frac{r_{g P}}{r}-\frac{a^{2}}{r_{g P} r}\right) c^{2} d t^{2}-\left(1-\frac{r_{g P}}{r}\right)^{-1}\left(1-\frac{a^{2}}{r_{g P} r}\right)^{-1} d r^{2}- \\
& -\left(r^{2}+a^{2}+\frac{a^{2} r_{g P}}{r}+\frac{a^{4}}{r_{g P} r}\right) d \varphi^{2}+\frac{2 a}{r}\left(r_{g E}+\frac{a^{2}}{r_{g P}}\right) d \varphi d t,
\end{aligned}
$$

In contrast to (5), it is clearly seen here that growth of $a$ leads to a decrease in $g_{00}$, and hence makes stronger the gravity of the source.

In the standard form of the Kerr metric (4), the contributions to $g_{00}$, which explicitly depend on $a$, fall off at large distances as $1 / r^{2}$, and therefore neglecting them with respect to the terms of order $1 / r$ gives $g_{00}(r, \theta) \rightarrow 1-r_{g E} / r$, i.e. $g_{00}$ asymptotically turns into the Schwarzschild metric with the total mass of the source $\bar{M}(a)$ :

$$
r_{g E}=2 G \bar{M}(a) / c^{2} .
$$

The rotational energy also contributes to the total mass $\bar{M}(a)$ and for this reason $\bar{M}(a)$ depends on $a$. Introducing the mass $M$ associated with $r_{g P}$ and using (8) we find: 


$$
r_{g P}=\frac{2 G M}{c^{2}}, \quad \bar{M}(a)=M+\frac{c^{4} a^{2}}{4 G^{2} M} .
$$

It can be seen here that, since $\bar{M}(a) \rightarrow M$ at $a \rightarrow 0$, here $M$ is a mass of the source without rotational energy.

Thus, the standard form of the Kerr metric (4) has the disadvantage that one of the two parameters included in it, $r_{g E}$, in fact, depends on the second, $a$, in the form (8). As can be seen from the metrics at the pole and equator, to eliminate this disadvantage, $r_{g E}$ in the Kerr metric can be expressed in terms of $r_{g P}$ according to (8). Making this substitution in the general case (4), we obtain the modified form of the Kerr metric:

$$
\begin{aligned}
& d s^{2}=\frac{r^{2}}{\rho^{2}}\left[1-\frac{r_{g P}}{r}-\frac{a^{2}}{r_{g P} r}\left(1-\frac{r_{g P}}{r} \cos ^{2} \theta\right)\right] c^{2} d t^{2}-\frac{\rho^{2}}{\Delta} d r^{2}-\rho^{2} d \theta^{2}- \\
& -\left[r^{2}+a^{2}+\frac{r a^{2}}{\rho^{2}}\left(r_{g P}+\frac{a^{2}}{r_{g P}}\right) \sin ^{2} \theta\right] \sin ^{2} \theta d \varphi^{2}+\frac{2 r a c}{\rho^{2}}\left(r_{g P}+\frac{a^{2}}{r_{g P}}\right) \sin ^{2} \theta d \varphi d t
\end{aligned}
$$

where $\Delta$ is given in (9). This metric contains two independent parameters $a$ and $r_{g P}$.

The gravitational radius $r_{g \theta}$, at which vanishes $g_{00}(r, \theta)$, is expressed in terms of the independent parameters as:

$$
r_{g \theta}=\frac{1}{2}\left(r_{g P}+\frac{a^{2}}{r_{g P}}\right)+\sqrt{\frac{1}{4}\left(r_{g P}+\frac{a^{2}}{r_{g P}}\right)^{2}-a^{2} \cos ^{2} \theta} .
$$

At the pole this gives $r_{g P}$, at the equator $r_{g E}$, and in the general case $r_{g \theta}$ describes the surface of the frozar with angular momentum as an oblate spheroid up to a small correction (Appendix A). Its surface area is, therefore,

$$
S=2 \pi r_{g E}^{2}+\pi \frac{r_{g P}^{2}}{e} \ln \frac{1+e}{1-e}, \quad e^{2}=1-\frac{r_{g P}^{2}}{r_{g E}^{2}},
$$

where $e$ is the eccentricity. In terms of the independent parameter $r_{g P}$, we have:

$$
\begin{gathered}
S=2 \pi r_{g P}^{2}\left(1+\frac{a^{2}}{r_{g P}^{2}}\right)\left[1+\frac{a^{2}}{r_{g P}^{2}}+\frac{r_{g P} / 2 a}{\sqrt{2+a^{2} / r_{g P}^{2}}} \ln B\right], \\
B=\frac{1+e}{1-e}=1+\frac{2 a^{2}}{r_{g P}^{2}}\left(2+\frac{a^{2}}{r_{g P}^{2}}\right)+\frac{2 a}{r_{g P}}\left(1+\frac{a^{2}}{r_{g P}^{2}}\right) \sqrt{2+\frac{a^{2}}{r_{g P}^{2}}}
\end{gathered}
$$

At $r>r_{g P}$, the metric (14) has no singularities and it is clearly seen in it that increasing of $a$ leads to decreasing of $g_{00}$, which means stronger gravity of the such source due to the contribution of the rotational energy. As a result, redshifts, the mean radii of orbits and shadow in the field of a rotating source increase with increasing $a$. The corresponding formulas and diagrams are presented in the next section. 
However, in the black hole theory, at studying the dependence of the observable effects on $a$, the parameter $r_{g E}$ was assumed to be constant $r_{g E}=c o n s t$, which led to the unphysical consequence that the growth of $a$, i.e. adding positive rotational energy, weakens the gravity of the source and therefore reduces the observable effects of gravity [6,7].

\section{Some observable consequences: mean radii of orbits}

In the standard Kerr metric (4) with the depending on $a$ parameter $r_{g E}$ the equations of motion, integrals of motion, and expressions for the characteristic radii of orbits are well known [6,7]. To obtain similar expressions following from the modified Kerr metric for frozar (14), including the independent on $a$ parameter $r_{g P}$, it is sufficient to make the substitution (8) in the former expressions for the characteristic radii. For this reason, it is enough to present the results of such a substitution and curves of the obtained dependence on $a$.

Introducing the notation $a_{*} \equiv a / r_{g P}$, we write the expression (8) for $r_{g E}$ in the form:

$$
r_{g E}=r_{g P}\left(1+a_{*}^{2}\right)
$$

This gravitational radius at the equator grows as $a^{2}$ (Fig. ), whereas in the black hole theory it was considered as constant $[4,7]$.

In the frozar metric with angular momentum (14), the radii of the photon orbit $r_{p h \pm}$ in two directions of rotation on the orbit, as well as the mean value of these radii $r_{p h 0}$ (where the influence of the dragging effect is excluded) are equal (Fig. 3):

$$
\begin{aligned}
& r_{p h \mp}=r_{g P}\left(1+a_{*}^{2}\right)\left\{1+\cos \left[\frac{2}{3} \arccos \left(\mp \frac{2 a_{*}}{1+a_{*}^{2}}\right)\right]\right\}, \\
& r_{p h 0}=\frac{1}{2}\left(r_{p h+}+r_{p h-}\right) .
\end{aligned}
$$

At $a=r_{g P} / 2, a_{*}=1 / 2$, for the upper sign (co-rotation) we have $r_{p h-} \simeq 1.13 r_{g P}$, for the lower sign (counter-rotation) $r_{p h+} \simeq 2.39 r_{g P}$, and their mean value is $r_{p h 0} \simeq 1.76 r_{g P}$. They are larger than for the black holes (Fig. 4): $r_{p h-} \simeq 0.5 r_{g P}, r_{p h+} \simeq 2 r_{g P}$ and $r_{p h 0} \simeq 1.25 r_{g P}$.

The radii of marginally bound orbits $r_{m b \pm}$ and $r_{m b 0}$ are given by the formulas (Fig. 5):

$$
\begin{aligned}
r_{m b \mp} & =r_{g P}\left(1+a_{*}^{2}\right)\left(1 \mp \frac{2 a_{*}}{1+a_{*}^{2}}+\sqrt{1 \mp \frac{2 a_{*}}{1+a_{*}^{2}}}\right), \\
r_{m b 0} & =\frac{1}{2}\left(r_{m b-}+r_{m b+}\right) .
\end{aligned}
$$

At $a=r_{g P} / 2, a_{*}=1 / 2$, we have $r_{p h-}=r_{g P} \cdot(3+\sqrt{5}) / 4 \simeq 1.31 r_{g P}$ (co-rotation), $r_{p h+}=r_{g P} \cdot(7+3 \sqrt{5}) / 4 \simeq 3.43 r_{g P}$ (counter-rotation) and $r_{m b 0}=2.37 r_{g P}$. They are higher than in the black hole theory (Fig. 6): $r_{p h-} \simeq 0.5 r_{g P}, r_{p h+} \simeq 2.91 r_{g P}$ and $r_{m b 0}=1.71 r_{g P}$. 


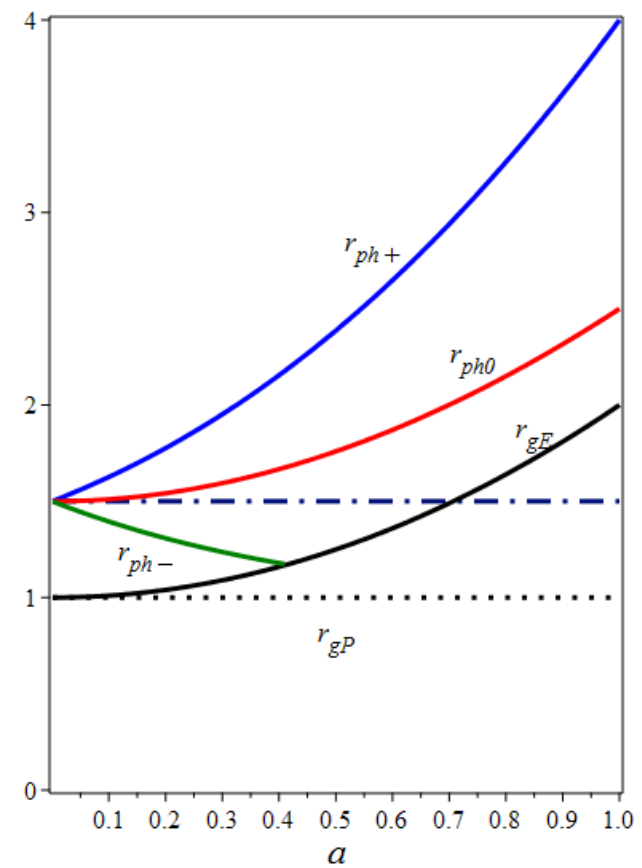

Fig. 3. Radii of photon orbits in two directions of motion $r_{p h \pm}$ and their mean value $r_{p h 0}$ in the modified Kerr metric for a frozar.

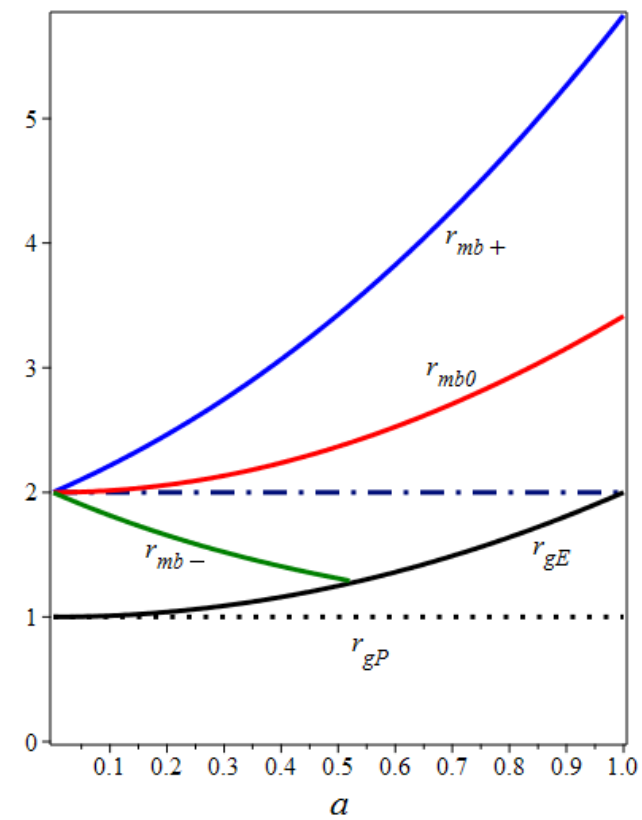

Fig. 5. Radii of the marginally bound orbits in two directions of motion $r_{m b \pm}$ and their mean value $r_{m b 0}$ in the modified Kerr metric for a frozar.

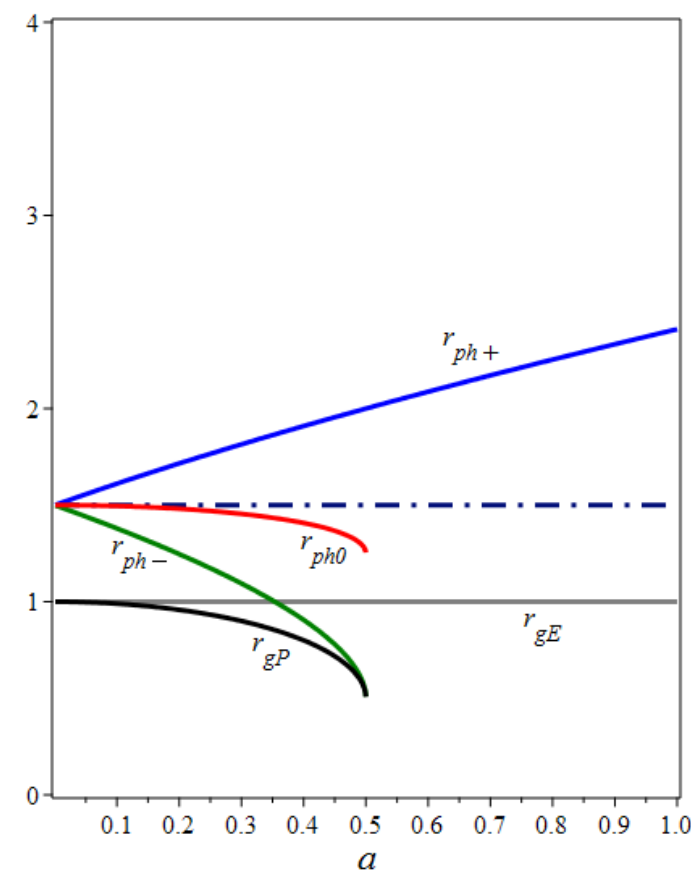

Fig. 4. Radii of photon orbits in two directions of motion $r_{p h \pm}$ and their mean value $r_{p h 0}$ in the standard Kerr metric for a black hole.

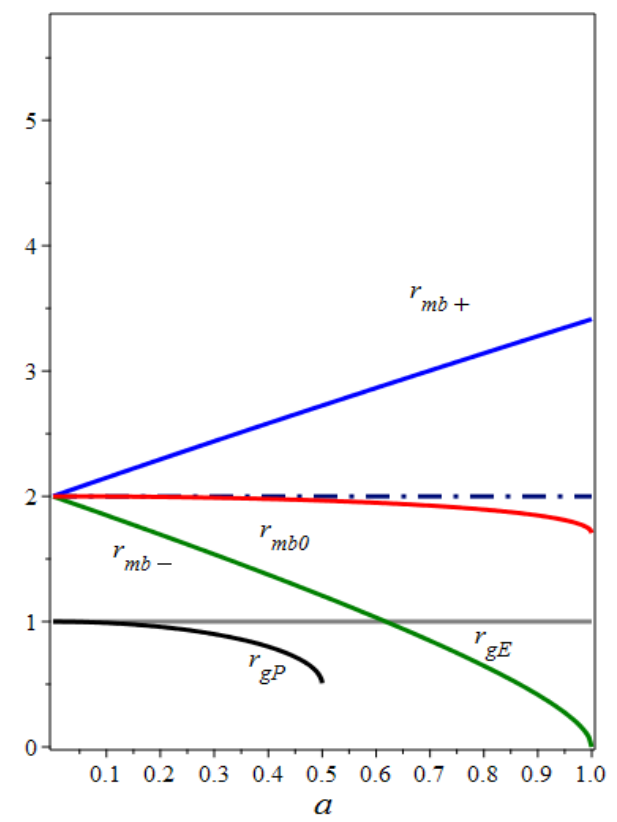

Fig. 6. Radii of the marginally bound orbits in two directions of motion $r_{m b \pm}$ and their mean value $r_{m b 0}$ in the standard Kerr metric for a black hole. 
The radii $r_{I S C O \pm}$ and $r_{I S C O 0}$ of the innermost stable circular orbit of a particle is equal to (Fig. 7):

$$
\begin{aligned}
r_{I S C O \mp}= & r_{g P}\left(1+a_{*}^{2}\right)\left\{\frac{3}{2}+Z_{2} \mp\left[\left(\frac{3}{2}-Z_{1}\right)\left(\frac{3}{2}+Z_{1}+2 Z_{2}\right)\right]^{1 / 2}\right\}, \\
r_{I S C O 0}= & \frac{1}{2}\left(r_{I S C O-}+r_{I S C O+}\right), \\
& Z_{1}=\frac{1}{2}\left\{1+\left(1-b^{2}\right)^{1 / 3}\left[(1+b)^{1 / 3}+(1-b)^{1 / 3}\right]\right\} \\
Z_{2}= & \left(Z_{1}^{2}+3 b^{2} / 4\right)^{1 / 2}, b=\frac{2 a_{*}}{1+a_{*}^{2}}
\end{aligned}
$$

At $a=r_{g P} / 2, a_{*}=1 / 2$, we have $r_{I S C O-} \simeq 2.25 r_{g P}$ for co-rotation, and $r_{I S C O+} \simeq 5.99 r_{g P}$ for counter-rotation and the mean is $r_{I S C O O} \simeq 4.12 r_{g P}$. These values differ significantly from the black hole theory ones (Fig. 8): $r_{I S C O-} \simeq 0.5 r_{g P}, r_{I S C O+} \simeq 4.5 r_{g P}$ and $r_{I S C O 0} \simeq 2.5 r_{g P}$.

Thus, with growth $a$, the gravitational radius at the pole practically does not change: $r_{g P}=$ const, while the expressions (8), (19)-(22) show that the radii $r_{g E}, r_{p h}, r_{m b}$ and $r_{I S C O}$ are multiplied to the additional factor growing as $a^{2}$. The corresponding curves are shown in Figs. 3,5,7.

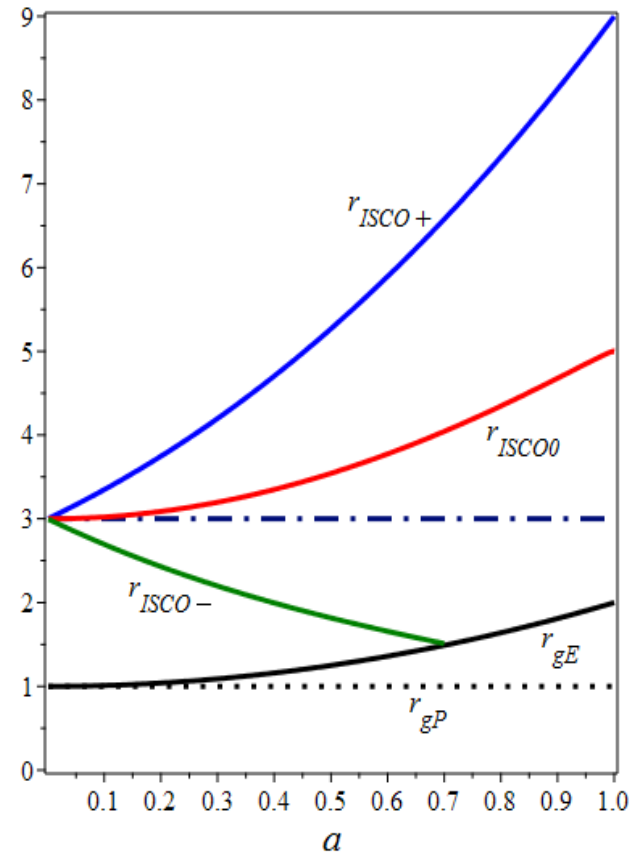

Fig. 7. Radii of the innermost stable circular orbits $r_{I S C O \pm}$ in two directions of motion and their mean $r_{I S C O O}$ in the modified Kerr metric for a frozar.

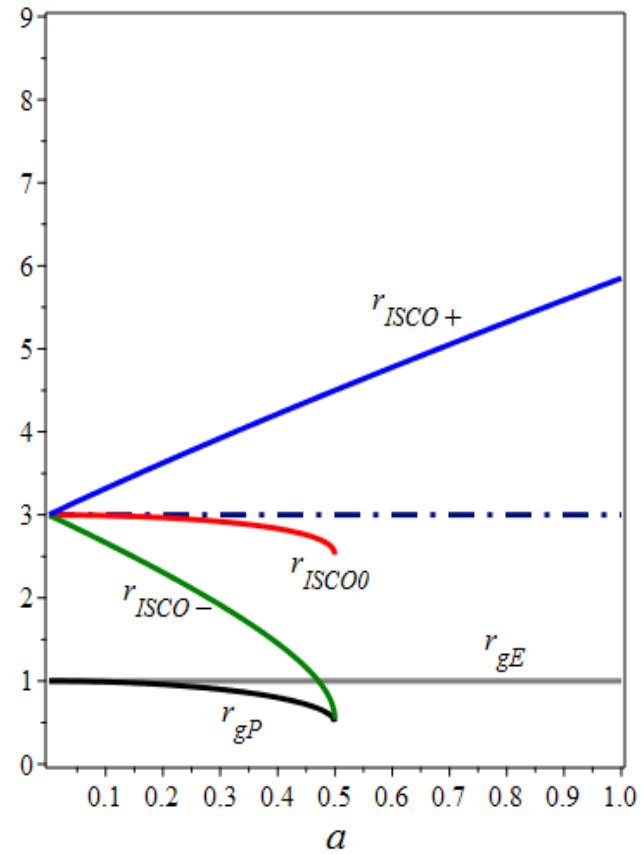

Fig. 8. Radii the innermost stable circular orbits $r_{I S C O \pm}$ in two directions of motion and their mean $r_{I S C O O}$ in the standard Kerr metric for a black hole. 
The corresponding predictions of the black hole theory [6,7] are also shown in Figs. 4,6,8. These predictions were made under the erroneous assumption that at growing $a$ the value $r_{g E}$ remains constant: $r_{g E}=$ const . As a result, it was concluded that an increase in the angular momentum of the source decreases the gravitational radius at the pole, the mean radii of the particles' orbits, and also decreases the mean size of the shadow at the equator.

As it was shown, the modified Kerr metric for frozar with the correct dependence $r_{g E}(a)$ excludes such erroneous predictions of the black hole theory.

\section{A modified form of the Kerr-Newman metric for a charged frozar with angular momentum}

For a point source (black hole) with angular momentum $J=M a c$ and charge $Q$ (Fig. 9) the standard Kerr-Newman metric [4] follows from the standard Kerr metric (4) at the replacement $r_{g E} r \rightarrow \bar{r}_{g E} r-Q^{2}$. This means that, at determining the metric at a point $r$, from the total mass at infinity $\bar{M}(a)$, including a mass-equivalent of the total energy of the electric field, the gravitationally inactive part of this energy outside the sphere of radius $r$ is subtracted (see [1]). The resulting line element has the form (see [7]):

$$
\begin{aligned}
d s^{2} & =\left(1-\frac{\bar{r}_{g E} r-Q^{2}}{\rho^{2}}\right) c^{2} d t^{2}-\frac{\rho^{2}}{\Delta_{Q}} d r^{2}-\rho^{2} d \theta^{2}+\frac{2 a c\left(\bar{r}_{g E} r-Q^{2}\right)}{\rho^{2}} \sin ^{2} \theta d \varphi d t- \\
& -\left(r^{2}+a^{2}+\frac{a^{2}\left(\bar{r}_{g E} r-Q^{2}\right)}{\rho^{2}} \sin ^{2} \theta\right) \sin ^{2} \theta d \varphi^{2}
\end{aligned}
$$

where $\bar{r}_{g E}$ is the gravitational radius of a charged source at the equator and:

$$
\Delta_{Q}(r)=r^{2}-\bar{r}_{g E} r+a^{2}+Q^{2} .
$$

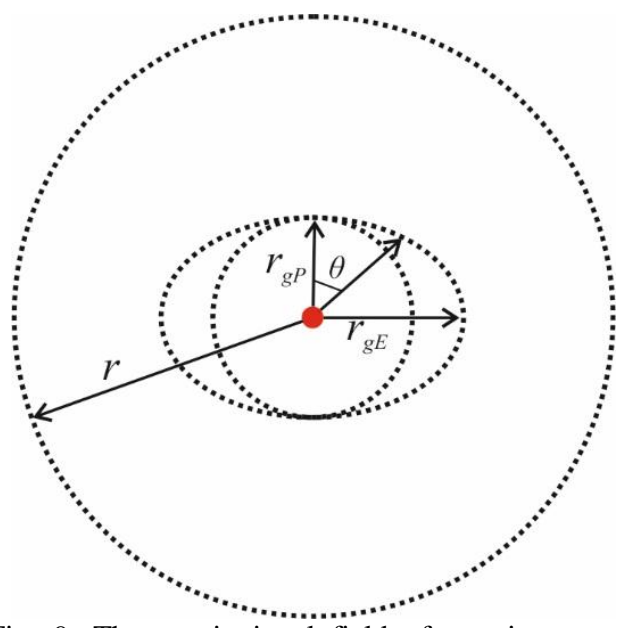

Fig. 9. The gravitational field of a point source (black hole) with charge and angular momentum. The equipotential surface of the electric field is spherically symmetric with radius $r$.

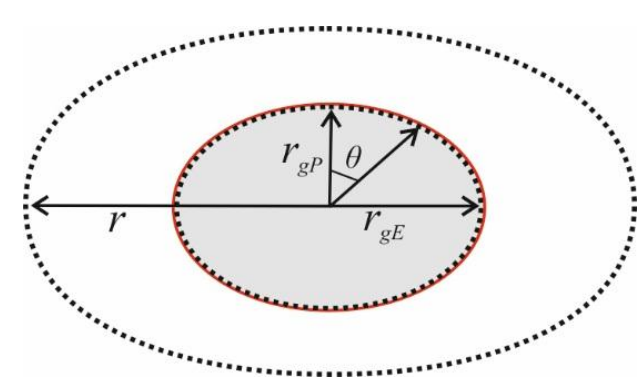

Fig. 10. Frozar with angular momentum and the surface charge. The surface freezes above the gravitational radius as an oblate ellipsoid. The equipotential surface of the electric field is flattened over the poles due to due to the higher surface charge density at the equator. 
At the equator, where $\theta=\pi / 2, \rho^{2}=r^{2}$, the line element (23) has the form: sliding

$$
\begin{aligned}
d s^{2} & =\frac{\Delta_{Q}-a^{2}}{r^{2}} c^{2} d t^{2}-\frac{r^{2}}{\Delta_{Q}} d r^{2}+\frac{2 a c}{r}\left(\bar{r}_{g E}-\frac{Q^{2}}{r}\right) d \varphi d t- \\
& -\left[r^{2}+a^{2}+\frac{a^{2}}{r}\left(\bar{r}_{g E}-\frac{Q^{2}}{r}\right)\right] d \varphi^{2},
\end{aligned}
$$

At the pole, where $\theta=0, \rho^{2}=r^{2}+a^{2}$, the line element (23) takes a simple form:

$$
d s^{2}=\frac{\Delta_{Q}}{r^{2}+a^{2}} c^{2} d t^{2}-\frac{r^{2}+a^{2}}{\Delta_{Q}} d r^{2} .
$$

In the frozar theory, the collapse of a real star with angular momentum and charge leads to the formation of a frozar with a frozen structure, the charged ellipsoidal surface of which freezes above the gravitational radius $r_{g \theta}$ (Fig. 10). Like a neutral frozar with angular momentum, the gravitational radius at the pole $r_{g P}$ follows from the condition:

$$
g_{00}\left(r_{g P}, 0\right)=\frac{\Delta_{Q}\left(r_{g P}\right)}{r_{g P}^{2}+a^{2}}=0, \quad \Delta_{Q}\left(r_{g P}\right)=r_{g P}^{2}-\bar{r}_{g E} r_{g P}+a^{2}+Q^{2}=0 .
$$

Dividing the expression for $\Delta_{Q}\left(r_{g P}\right)$ by $r_{g P}$, we can express $\bar{r}_{g E}$ in terms of $r_{g P}$ :

$$
\bar{r}_{g E}=r_{g P}+\frac{a^{2}+Q^{2}}{r_{g P}} .
$$

The expression for $\Delta_{Q}(r)$ in (24) can be written in terms of the independent parameter $r_{g P}$ :

$$
\Delta_{Q}(r)=r^{2}-\left(r_{g P}+\frac{a^{2}+Q^{2}}{r_{g P}}\right) r+a^{2}+Q^{2}=r^{2}\left(1-\frac{r_{g P}}{r}\right)\left(1-\frac{a^{2}+Q^{2}}{r_{g P} r}\right) .
$$

and, by substituting this and (28) into (26), we come to the modified line element at the pole:

$$
d s^{2}=\frac{r^{2}}{\bar{\rho}^{2}}\left(1-\frac{r_{g P}}{r}\right)\left(1-\frac{a^{2}+Q^{2}}{r_{g P} r}\right) c^{2} d t^{2}-\frac{\bar{\rho}^{2}}{r^{2}}\left(1-\frac{r_{g P}}{r}\right)^{-1}\left(1-\frac{a^{2}+Q^{2}}{r_{g P} r}\right)^{-1} d r^{2}
$$

where $\bar{\rho}^{2}=r^{2}+a^{2}$. Here it is clear the role of $r_{g P}$ as the gravitational radius at the pole. By substituting (28) into (25), we obtain the modified line element at the equator:

$$
\begin{aligned}
& d s^{2}=\left[\left(1-\frac{r_{g P}}{r}\right)\left(1-\frac{a^{2}+Q^{2}}{r_{g P} r}\right)-\frac{a^{2}}{r^{2}}\right] c^{2} d t^{2}-\left(1-\frac{r_{g P}}{r}\right)^{-1}\left(1-\frac{a^{2}+Q^{2}}{r_{g P} r}\right)^{-1} d r^{2}- \\
& -\left[r^{2}+a^{2}+\frac{a^{2}}{r}\left(r_{g P}+\frac{a^{2}+Q^{2}\left(1-r_{g P} / r\right)}{r_{g P}}\right)\right] d \varphi^{2}+ \\
& +\frac{2 a c}{r}\left(r_{g P}+\frac{a^{2}+Q^{2}\left(1-r_{g P} / r\right)}{r_{g P}}\right) d \varphi d t,
\end{aligned}
$$

or 


$$
\begin{aligned}
d s^{2} & =\frac{\Delta_{Q}-a^{2}}{r^{2}} c^{2} d t^{2}-\frac{r^{2}}{\Delta_{Q}} d r^{2}+\frac{2 a c}{r}\left(r_{g P}+\frac{a^{2}+Q^{2}\left(1-r_{g P} / r\right)}{r_{g P}}\right) d \varphi d t- \\
& -\left[r^{2}+a^{2}+\frac{a^{2}}{r}\left(r_{g P}+\frac{a^{2}+Q^{2}\left(1-r_{g P} / r\right)}{r_{g P}}\right)\right] d \varphi^{2} .
\end{aligned}
$$

Unlike (25), it is clearly seen here that increasing of $a$ and/or $Q$, unchanging $r_{g P}$, leads to a decrease in $g_{00}$, and hence makes stronger the gravity of the source.

In the Kerr-Newman metric (23), at large distances $g_{00}(r, \theta) \rightarrow 1-\bar{r}_{g E} / r$ and $g_{00}$ in asymptotics turns into the Schwarzschild metric with total mass $\bar{M}(a, Q)$ :

$$
\bar{r}_{g E}=2 G \bar{M}(a, Q) / c^{2} .
$$

The total mass of the source $\bar{M}(a, Q)$ includes the energies of rotation and the electric field and for this reason it depends on $a$ and $Q$. Introducing the mass $M$ associated with the gravitational radius $r_{g P}$ of the neutral non-rotating matter and taking into account (28), we find

$$
r_{g P}=\frac{2 G M}{c^{2}}, \quad \bar{M}(a, Q)=M+\frac{c^{4}\left(a^{2}+Q^{2}\right)}{4 G^{2} M} .
$$

Since $\bar{M}(a, Q) \rightarrow M$ at $a \rightarrow 0$ and $Q \rightarrow 0$, we see that $M$ really is the mass of the source's without the energies of rotation and the electric field. Therefore, $r_{g P}$ is a parameter, independent of $a$ and $Q$, and characterizing the contribution of a neutral non-rotating matter, while $\bar{r}_{g E}$ is a dependent parameter growing quadratically with growth $a$ and/or $Q$.

Notice, that an attempt to take into account such a dependence in [8], based on heuristic arguments, was not taken into account in the metric (23) itself. The reason for this was not only that this dependence was unreasonably complicated, but also that in the black hole theory there is no physically correct definition of the mass $M[1]$ and the contribution of the electric field. For this reason, it was determined through the dependent parameter $\bar{M}(a, Q)$, which complicated the situation, leading to a set of non-physical consequences.

Thus, in the standard form of the Kerr-Newman metric (23), one of the three parameters, $\bar{r}_{g E}$, depends on the other two parameters $a$ and $Q$, and to correct this weakness, it is sufficient to express $\bar{r}_{g E}$ in terms of $r_{g P}$ as in (28). Substituting (28) into (23) and using (29), we arrive at the modified Kerr-Newman metric with three independent parameter $a, Q$ и $r_{g P}$ :

$$
\begin{aligned}
& d s^{2}=\frac{\Delta_{Q}-a^{2} \sin ^{2} \theta}{\rho^{2}} c^{2} d t^{2}-\frac{\rho^{2}}{\Delta_{Q}} d r^{2}-\rho^{2} d \theta^{2}- \\
& -\left[r^{2}+a^{2}+\frac{r a^{2}}{\rho^{2}}\left(r_{g P}+\frac{a^{2}+Q^{2}\left(1-r_{g P} / r\right)}{r_{g P}}\right) \sin ^{2} \theta\right] \sin ^{2} \theta d \varphi^{2}+ \\
& +\frac{2 r a c}{\rho^{2}}\left(r_{g P}+\frac{a^{2}+Q^{2}\left(1-r_{g P} / r\right)}{r_{g P}}\right) \sin ^{2} \theta d \varphi d t .
\end{aligned}
$$


The gravitational radius $r_{g \theta}$, at which vanishes $g_{00}(r, \theta)$, is expressed in terms of the independent parameters as:

$$
r_{g \theta}=\frac{1}{2}\left(r_{g P}+\frac{a^{2}+Q^{2}}{r_{g P}}\right)+\sqrt{\frac{1}{4}\left(r_{g P}+\frac{a^{2}+Q^{2}}{r_{g P}}\right)^{2}-a^{2} \cos ^{2} \theta-Q^{2}} .
$$

At the pole this gives $r_{g P}$, and at the equator we get the gravitational radius $r_{g E}$ :

$$
r_{g E}=\frac{\bar{r}_{g E}}{2}+\sqrt{\frac{\bar{r}_{g E}^{2}}{4}-Q^{2}}=\frac{1}{2}\left(r_{g P}+\frac{a^{2}+Q^{2}}{r_{g P}}\right)+\sqrt{\frac{1}{4}\left(r_{g P}+\frac{a^{2}+Q^{2}}{r_{g P}}\right)^{2}-Q^{2}} .
$$

In the general case, $r_{g \theta}$ describes the surface of the charged frozar with angular momentum as an oblate spheroid (up to a very small correction), where its charge is located. The area we find from (50), where now (37) is given.

Since the surface charge density at the equator is higher than at the pole, this deforms the outer electric field of the frozar so that the equipotential surfaces are not just spheroidal, but will contracted at the poles (Fig. 10). These properties show the fundamental difference between the frozar field and the field of a point charge (black hole), in which the electric field, obviously, remains spherically symmetric (Fig. 9). The deformity of the frozar field can be detected by various effects depending on the configuration of the electric field of the frozar. The corresponding observational effects will be considered in more detail in subsequent publications and in [10].

At $r>r_{g P}$, the metric (35) has no singularities and it is clearly seen that an increase in $a$ and/or $Q$ decreases $g_{00}$ and therefore makes stronger the gravity of the source due to the contribution of the energies of rotation and the electric field. Therefore, redshifts, the mean radii of orbits and shadows increase with increasing $a$ and/or $Q$.

The previous predictions of the black hole theory for the dependence of the observable effects on the change $a$ or $Q$, made at a constant parameter $\bar{r}_{g E}$, should also be replaced by the predictions of the frozar theory taking into account this dependence correctly.

\section{Thermodynamics of frozars}

The change in the total energy of the frozar with the mass of matter $M$ (in the neutral and non-rotating state), charge $Q$ and angular momentum $J$ is given by the relation:

$$
d E=c^{2} d M+\Omega d J+\Phi d Q,
$$

where $\Omega, \Phi$ - angular velocity and electrostatic potential. This can be called the first law of thermodynamics of frozars.

Falling on the frozar of a particle with the opposite charge decreases the charge of the frozar $Q$, thus decreasing the energy of the electrostatic field. Frozar's angular momentum $J$ is reduced by any process in which angular momentum transfers a part of the rotational energy on particles at a sufficient distance (or when interacting with external fields). And vice versa, $Q$ and $J$ increase in similar processes with the opposite result. Thus, changes in $Q$ and $J$ can be carried out in a reversible way.

The situation is opposite for changes in $M$ that are practically irreversible. Due to the frozen nature of frozar, giant acceleration and redshift on its surface, a decrease in its rest energy (or mass $M$ ) by exiting particles (both non-zero mass and radiation) with appreciable energy 
from it has a negligible probability. Therefore, an increase in mass over time occurs with an overwhelming probability due to the fall of matter and radiation from outside:

$$
\frac{d M}{d t} \geq 0 \text {. }
$$

This, accordingly, is the second law of thermodynamics of frozars, which means practically non-decreasing "bare" mass. Although this is an analogue of the non-decreasing entropy, here we are not talking about a direct analogy, and the possible introduction of both entropy and a certain temperature is purely formal.

And, finally, the third law follows from the fact that the total energy of frozar $E$, including the rest energy $E_{0}=M c^{2}$ and the energies of rotation and the electric field, can only decrease until $E_{0}$ as a background state, when the frozar loses both angular momentum and charge, becoming Schwarzschildean (spherical and neutral):

$$
E \geq M c^{2}
$$

At the same time, the "evaporation" of a neutral spherical frozar due to the creation of virtual pairs, when one of them leaves the frozar, is practically impossible. This is due to the fact that both particles in the pair have positive energy both inside and in the vicinity of the frozar. The remaining particle can only increase the energy of the frozar, in contrast to the wrong statement of the black hole theory.

Thus, the former effects of the black hole theory (evaporation, extraction of rotational energy from the ergosphere) (see [7]) were unphysical, since frozars have no singularities, horizons and ergosphere and energies of particles remain positive everywhere $[9,10]$.

\section{Conclusion}

The standard form of the metric outside a rotating source, in both neutral (Kerr) and charged (Kerr-Newman) cases, includes two or three parameters, one of which, the gravitational radius at the equator $\bar{r}_{g E}$, is dependent. But the metric can be reduced to a form with independent parameters, and in the article this form of the metric is obtained in both cases. This new metric includes the gravitational radius of the neutral non-rotating matter $r_{g P}$.

This made it possible to exclude the previous unphysical predictions of the black hole theory, that an increase in the specific angular momentum $a$ and/or charge $Q$ weakens gravity outside the source, with the prediction of weakening the physical effects of gravity [6,7].

Instead, the frozar theory, from which this form of the metric follows naturally, predicts the opposite, that an increase in $a$ and/or $Q$, adding positive energy of rotation and/or electric field, makes stronger gravity of the source with a corresponding increasing the physical effects of gravity.

A similar procedure is applicable to all other metrics describing the contribution to the field of compact sources of various fields and other factors. An example of modification of the NUT metric is presented in the Appendix. Other applications of the method and a more detailed discussion of the consequences of the modified form of the Kerr and Kerr-Newman metrics will be considered in following articles and in the book [10]. 


\section{Appendix.}

A. Formation of an ellipsoidal frozar at freezing of the rotating star's surface

In the Kerr metric, as in any gravitational field, the proper time $\tau$ of a particle at rest at $(r, \theta, \varphi)=$ const. is defined as:

$$
d \tau^{2}=g_{00} d t^{2} .
$$

At $r \rightarrow r_{g \theta}$ this proper time freezes relative to world time $t$, which is purely gravitational time dilation. The proper time $\tau$ of the particle's comoving surface with a local velocity $\mathbf{v}$ is slowed down relatively $t$ both gravitationally and kinematically [9,10]:

$$
\begin{gathered}
d \tau^{2}=g_{00} d t^{2}\left(1-\mathbf{v}^{2} / c^{2}\right), \\
\mathbf{v}^{2}=g_{00}^{-1}\left\{\frac{\rho^{2}}{\Delta} v_{r}^{2}+\rho^{2} v_{\theta}^{2}+\left[\left(r^{2}+a^{2}+\frac{r_{g E} r a^{2}}{\rho^{2}} \sin ^{2} \theta\right) \Omega-\frac{2 r_{g E} r a c}{\rho^{2}}\right] \Omega \sin ^{2} \theta\right\},
\end{gathered}
$$

where $v_{r}=d r / d t, v_{\theta}=d \theta / d t, v_{\varphi}=d \varphi / d t=\Omega$.

At the pole $\theta=\pi / 2, \rho=r$, we have:

$$
\begin{gathered}
d \tau^{2}=\frac{1-a^{2} / r_{g P} r}{1+a^{2} / r^{2}}\left(1-\frac{r_{g P}}{r}\right)\left(1-\frac{\mathbf{v}^{2}}{c^{2}}\right) d t^{2}, \\
|\mathbf{v}|=\left[\frac{1-a^{2} / r_{g P} r}{1+a^{2} / r^{2}}\left(1-\frac{r_{g P}}{r}\right)\right]^{-1} v_{r}
\end{gathered}
$$

Thus, for $r \rightarrow r_{g P}$ and $|\mathbf{v}| \rightarrow c$ for the radial velocity $v_{r}$, we obtain:

$$
v_{r} \rightarrow \frac{1-a^{2} / r_{g P}^{2}}{1+a^{2} / r_{g P}^{2}}\left(1-\frac{r_{g P}}{r}\right) \rightarrow 0
$$

i.e. $v_{r} \rightarrow 0$, and the surface freezes above the gravitational radius $r_{g P}$.

On the equatorial plane $\theta=\pi / 2, \rho=r$, we have:

$$
\begin{gathered}
d \tau^{2}=d t^{2}\left(1-\frac{r_{g E}}{r}\right)\left(1-\frac{\mathbf{v}^{2}}{c^{2}}\right), \\
\mathbf{v}^{2}=\left(1-\frac{r_{g E}}{r}\right)^{-1}\left[\frac{r^{2}}{\Delta} v_{r}^{2}+\left(r^{2}+a^{2}+\frac{a^{2} r_{g E}}{r}\right) \Omega^{2}-\frac{2 r_{g E} a c}{r} \Omega\right] .
\end{gathered}
$$

At $\mathbf{v}^{2} \rightarrow c$ we have from (48) following relations:

$$
\frac{r^{2} v_{r}^{2}}{\Delta}-\frac{2 r_{g E} a c}{r} \Omega+\left(r^{2}+a^{2}+\frac{a^{2} r_{g E}}{r}\right) \Omega^{2} \rightarrow\left(1-\frac{r_{g E}}{r}\right) .
$$

Therefore, at $r \rightarrow r_{g E}$ we have: 


$$
\left[\left(r_{g E}^{2}+2 a^{2}\right)|\Omega| \mp 2 a c\right]|\Omega|+\frac{r_{g E}^{2}}{a^{2}} v_{r}^{2} \rightarrow 0 .
$$

Here, in any case $v_{r}^{2} \rightarrow 0$, and as for $\Omega$, at first glance it seems that the term $\mp 2 a c$ with a minus sign can give the solution $|\Omega|=2 a c /\left(r_{g E}^{2}+2 a^{2}\right)>0$, while the plus sign allows only the solution $|\Omega| \rightarrow 0$. Since space is isotropic, a simple change in the sign of the angular velocity cannot lead to a qualitative change in the state of the object, and therefore only the first solution with $|\Omega| \rightarrow 0$ is physically admissible. Thus, as the equator approaches $r_{g E}$, the entire surface practically freezes in terms of $t$, the inner layers also freeze, and the star passes into a frozar state with angular momentum.

Notice that the angular momentum of a rotating star is conserved, and its external field remains the same as before freezing, since the asymptotical freezing does not mean an absolute stopping. The local speed of particles on the frozar's surface is close to the light speed, while for an external static frame of reference, frozar looks almost like a static ellipsoidal star.

\section{B. Modified NUT metric}

The standard NUT metric has the form [5]:

$$
\begin{gathered}
d s^{2}=\Delta\left(c d t+4 n \sin ^{2} \frac{\theta}{2} \cdot d \varphi\right)^{2}-\frac{d r^{2}}{\Delta}-\left(r^{2}+n^{2}\right)\left(d \theta^{2}+\sin ^{2} \theta d \varphi^{2}\right) \\
\Delta=1-\frac{\bar{r}_{g} r+2 n^{2}}{r^{2}+n^{2}} .
\end{gathered}
$$

The gravitational radius $r_{g}$ is determined by the condition $g_{00}\left(r_{g P}, 0\right) \sim \Delta=0$, which gives:

$$
r_{g}^{2}-\bar{r}_{g} r_{g}-n^{2}=0 \text {. }
$$

Dividing this equality by $r_{g}$, we obtain the expression $\bar{r}_{g}$ through $r_{g}$ :

$$
\bar{r}_{g}=r_{g}-\frac{n^{2}}{r_{g}}, \quad n \geq r_{g} .
$$

Substituting this into (52), we can express $\Delta$ in terms of $r_{g}$ :

$$
\Delta=\frac{1+n^{2} / r r_{g}}{1+n^{2} / r^{2}}\left(1-\frac{r_{g}}{r}\right)
$$

Thus, in the NUT metric there is only one gravitational radius $r_{g}$, which simplifies the structure of space-time around such a source. At $n \neq 0$ there are conditions $r>r_{g}>\bar{r}_{g}$. 


\section{References}

1. Zakir Z. (2022) Quant. and Grav. Phys., 3:016-7677.

2. Kerr R. (1963) Phys. Rev. Lett., 5, 1.

3. Boyer R., Lindquist R. (1967) J. Math. Phys. 8, 265.

4. Newman E., Couch E., Chinnaparet K. et al (1965) J. Math. Phys. 6, 918.

5. Newman E., Tamburino L., Unti T. (1963) J. Math. Phys. 4(7), 915.

6. Bardeen J., Press W., Teukolsky S. (1972) Astrophys. J. 178, 347.

7. Bambi C. (2017) Black Holes: A Lab. for Testing Strong Gravity. Springer.

8. Christodoulou D., Ruffini R. (1971) Phys. Rev., D4, 3552.

9. Zakir Z. (2018) Astr. \& Space Sci. 363: 30; (2020) Quant. \& Grav. Phys. 1:006-7132; 1:007-7133

10. Zakir Z. (2022) Theory of Frozars. Relativistic Collapse of Stars, CTPA, T. 